\title{
The Effectiveness of a Group Motor Skill Intervention Program in Children with Developmental Coordination Disorder: Program Frequency Matters.
}

\author{
Priscila M. Caçola \\ University of Texas at Arlington, cacola@uta.edu \\ Melvin Ibana \\ University of Texas at Arlington \\ Michael Romero \\ University of Texas at Arlington \\ Jennifer Chuang \\ University of Texas at Arlington
}

Follow this and additional works at: https://nsuworks.nova.edu/ijahsp

Part of the Medicine and Health Sciences Commons

\section{Recommended Citation}

Caçola PM, Ibana M, Romero M, Chuang J. The Effectiveness of a Group Motor Skill Intervention Program in Children with Developmental Coordination Disorder: Program Frequency Matters.. The Internet Journal of Allied Health Sciences and Practice. 2016 Jan 01;14(1), Article 4.

This Manuscript is brought to you for free and open access by the College of Health Care Sciences at NSUWorks. It has been accepted for inclusion in Internet Journal of Allied Health Sciences and Practice by an authorized editor of NSUWorks. For more information, please contact nsuworks@nova.edu. 


\title{
The Effectiveness of a Group Motor Skill Intervention Program in Children with Developmental Coordination Disorder: Program Frequency Matters.
}

\begin{abstract}
Purpose: The purpose of this study was to investigate the effects of two group programs on visualmotor integration, motor proficiency, gross-motor skills, and parental perception of motor difficulties in children with developmental coordination disorder (DCD). We compared whether the frequency of two programs (10 one hour sessions - once/week for 10 weeks verses 24 one hour sessions - twice/week for 12 weeks) produced different changes in motor skills. Methods: Children in the two programs were assessed before and after each program with the MABC-2, DCD-Q, BOT-2, Beery-VMI-6, and the TGMD-2 assessments. The emphasis of the programs was on group activities that required collaboration while performing skills that utilized upper-body coordination, balance, and fine-motor abilities. Results: After the 24-session intervention program, results revealed significant improvements in the Manual Coordination and Total Score of the BOT-2 (ps $<</ e m>.05)$, and parents reported significantly higher scores for all categories of the $D C D-Q$ (ps $<</ \mathrm{em}>.05$ ). No significant changes were observed after the 10-session once/ week intervention program. Conclusions: We conclude that a (24 one-hour sessions- twice a week for 12 weeks) group intervention program can be an effective intervention method for improvement of motor skills in children with DCD. We recommend that allied health professionals consider the group intervention as a feasible way to promote more frequent sessions to children with DCD.
\end{abstract}

\section{Author Bio(s)}

- Priscila M. Caçola, PhD, is an Assistant Professor in the Department of Kinesiology and Director of the Developmental Motor Cognition Lab at the University of Texas at Arlington.

- Melvin Ibana has been at UTA since 2008. This is his last semester as an exercise science major. He has been a volunteer in the Developmental Motor and Cognition Lab since the beginning of 2012 and is now doing an internship with Little Mavs Movement Academy.

- Michael Romero is an undergraduate kinesiology major from El Paso, Texas. Following his mother's footsteps, Michael will pursue a master's in speech language pathology. He also hopes to follow his father's steps one day, by coaching track and field on the side.

- Jennifer Chuang is a post baccalaureate student with a degree in Business Management and professional experience in logistics. She is currently majoring in Biology and aspires to be a Pediatric Physical Therapist. Her other interests involve fitness, nutrition and travel. As a member of Team in Training she is raising money for Leukemia \& Lymphoma Society and training for a marathon. 


\title{
TIAHSP \\ The Internet Joưnal of Allied Health Sciences and Practice
}

Dedicated to allied health professional practice and education

Vol. 14 No. 1 ISSN 1540-580X

\section{The Effectiveness of a Group Motor Skill Intervention Program in Children with Developmental Coordination Disorder: Program Frequency Matters}

\author{
Priscila M. Cacola, PhD \\ Melvin Ibana \\ Michael Romero \\ Jennifer Chuang
}

University of Texas at Arlington

United States

\begin{abstract}
Purpose: The purpose of this study was to investigate the effects of two group programs on visual-motor integration, motor proficiency, gross-motor skills, and parental perception of motor difficulties in children with developmental coordination disorder (DCD). We compared whether the frequency of two programs (10 one hour sessions - once/week for 10 weeks verses 24 one hour sessions - twice/week for 12 weeks) produced different changes in motor skills. Methods: Children in the two programs were assessed before and after each program with the MABC-2, DCD-Q, BOT-2, Beery-VMI-6, and the TGMD-2 assessments. The emphasis of the programs was on group activities that required collaboration while performing skills that utilized upper-body coordination, balance, and fine-motor abilities. Results: After the 24-session intervention program, results revealed significant improvements in the Manual Coordination and Total Score of the BOT-2 ( $p s<.05)$, and parents reported significantly higher scores for all categories of the DCD-Q (ps <.05). No significant changes were observed after the 10-session once/week intervention program. Conclusions: We conclude that a (24 one-hour sessions- twice a week for 12 weeks) group intervention program can be an effective intervention method for improvement of motor skills in children with DCD. We recommend that allied health professionals consider the group intervention as a feasible way to promote more frequent sessions to children with DCD.
\end{abstract}

\section{INTRODUCTION}

Developmental Coordination Disorder (DCD) affects children with challenges in their motor coordination development despite their intelligence levels, and impacts about 5 to $6 \%$ of school-age children. ${ }^{1}$ While the degree of motor impairment varies, common symptoms of the disorder include marked delays in achieving motor milestones and clumsiness, and is normally associated with poor balance, coordination, and handwriting skills. ${ }^{2}$ Those difficulties, without accommodations, significantly interfere with activities of daily living or academic achievement. For this reason, children with DCD benefit from intervention focusing on developing skills and strategies to cope with the consequences associated with DCD. ${ }^{3} \mathrm{~A}$ meta-analysis by Smits-Engelsman et al pointed out that in general, intervention is shown to benefit motor performance of children with DCD over and above no intervention. ${ }^{4}$ Currently, however, the most effective frequency (as in number of sessions per week) and total number of sessions necessary to produce significant improvements in motor skills of children with DCD has yet to be identified.

Even though most therapy interventions designed for children with DCD are based and administered individually, a few studies have explored the effectiveness of group interventions for this population and reported mixed findings. A meta-analysis by Pless and Carlsson indicated that group-based therapy (effect size $=0.96$ ) may be more effective than individual-based therapy (effect size $=0.45$ ) for improvement of motor skills. ${ }^{5}$ Pless et al compared the results of a 10 -session group intervention for 5 - and 6 -yearolds with a control group who did not receive any intervention, with results indicating no differences between groups. ${ }^{6}$ However, when subgroups with different categories of motor difficulties were compared, significantly more children with borderline motor difficulties in the experimental compared to the control group improved after intervention. Hung \& Pang compared the effects of group-based to individual-based training on motor performance of young children with $\mathrm{DCD} .{ }^{7}$ The conditions were randomly

(c) The Internet Journal of Allied Health Sciences and Practice, 2016 
assigned and received the same intervention for 8 sessions, showing improvements after the intervention but no group differences, concluding that there is no benefit of individual intervention over group intervention. Dunford looked at a group of children who set their goals for a short-term with intense group intervention that showed significant improvements in motor function, indicating that a goal-oriented group intervention has potential as an effective intervention method. ${ }^{8}$

The previous articles support the notion that group-based interventions are effective for children with $D C D$, and might be considered a feasible, efficient, and more economical option for treatment. ${ }^{6}$ However, one interesting and common factor across the previous studies is the use of the Movement Assessment Battery for Children (M-ABC) test to detect possible changes in motor coordination after the intervention program. ${ }^{5-7}$ This test is designed primarily to help identify problems or delays in motor development and is considered a gold-standard assessment for diagnosis of Developmental Coordination Disorder. While one of the aims of the M$A B C$ test is to "measure change as a result of intervention", it is possible that the assessment is not sensitive enough to pick up all changes that might result from an intervention program. ${ }^{9}$ Consequently, a variety of assessments should be used. However, no studies have investigated the effect of group interventions using other motor assessments or more than one assessment at the same time.

Another factor observed with the previous studies was the amount or number of sessions utilized - most administered sessions once a week for 8 or 10 weeks and indicated slight improvements in motor performance. ${ }^{6,7} \mathrm{~A}$ more recent study combined eight 50-min sessions over 2 weeks and results indicated strong improvements in children's motor skills. ${ }^{8}$ While there is ambiguity in the results of group-based motor skill programs, it appears that frequency, duration, and intensity could also be one of the variables predicting effectiveness of this type of therapy. Research evidence regarding intensity, as in how often, how long, and the duration of an episode of physical therapy or occupational therapy is limited and difficult to generalize..$^{10}$ The present study focused on looking at potential differences between frequency and total number of sessions. The research questions were: Can a group intervention program improve several aspects of motor skills and parental perception of children with DCD? And if so, can the frequency of sessions be a factor in children's improvement? To that end, the effectiveness of two programs were tested - Program 1 with 24 sessions - twice/week of intervention, with a considerable number of sessions and more than twice as many sessions tested in previous studies, and Program 2, 10 sessions - once/week of intervention, with the same number of sessions as most previous studies. The same aspects of motor competence were applied in both programs, however, Program 2, due to the shorter number of sessions, had a combined focus, while Program 1 dedicated specific sessions to each one of the aspects trained.

Therefore, we compared the effects of two group intervention programs for children with DCD on different aspects of motor competence (visual-motor integration, motor proficiency, gross-motor skills), as well as parental perception of motor difficulties. We also compared whether frequency of the program (10 sessions - once/week verse 24 sessions - twice/week of intervention) produced significant changes in motor behavior. Both programs used activities that focused on group collaboration and exploration of at least one element of cooperation among two or more children, while performing tasks of upper-body coordination, balance, and fine-motor skills. Changes in visual-motor integration, motor proficiency, gross-motor skills, and parental perception were assessed before and after each program. The hypothesis was that children with DCD would improve more after participating in Program 1 (24 sessions), than in Program 2 (10 sessions). In other words, we expected that the frequency and the number of sessions of the group program would have a positive effect on motor skills of children with DCD.

\section{METHODS}

\section{Study Design}

This is a repeated measures study, with the same pre- and post-measurements taken before and after two intervention programs (Program 1 and Program 2) that differed in their number and frequency of sessions (Program 1: 24 sessions in 12 weeks; Program 2: 10 sessions in 10 weeks). A group setting was used for both intervention programs. The experimental protocol and consent form were approved by the Institutional Review Board (IRB) for the ethical treatment of human subjects. Families of children who met the DSM-5 Inclusion criteria for Developmental Coordination Disorder (see below) were contacted and told about the study, and upon agreement, an appointment was scheduled for the child and at least one guardian signed the consent forms and committed to program attendance. ${ }^{1}$ Participants were informed of the experimental procedures and voluntarily signed a consent form before participating in this study; children provided verbal consent after guardians signed the consent form.

\section{Participants}

A total of 14 children with DCD between 7 - and 12-years of age participated in the study; 8 were enrolled in Program 1 ( 2 females, 6 males) (24 one hour sessions- twice a week for 12 weeks), and 6 in Program 2 ( 1 female, 5 males) (10 one hour sessions- once a week for 10 weeks). Placement in programs was based on guardian and child availability - every guardian that replied to the study announcement was told of the two programs and the number of sessions, and was able to choose which one they wanted to be involved with. Qualification for DCD was based on the fit to the diagnostic criteria defined by the DSM-510: $(A)$ a score below

(C) The Internet Journal of Allied Health Sciences and Practice, 2016 
the 5th percentile on the Movement Assessment Battery for Children, 2e, MABC-2; (2) experience of motor coordination difficulties that had an impact on their daily function as assessed by a parental report (Developmental Coordination Disorder Questionnaire, DCD-Q); (C) elimination of another general medical condition such as cerebral palsy, hemiplegia or muscular dystrophy (report from pediatrician/parent). ${ }^{9,11}$ In addition, all children demonstrated at least "average" cognitive ability as measured by the Kaufmann Brief Intelligence Test - 2e. (KBIT-2).12

\section{Measurements}

A variety of assessments were chosen to detect changes in a wide range of motor competence of children. The Movement Assessment Battery for Children, 2e (MABC-2) is a product-oriented motor assessment used to detect motor impairments. This assessment has been used in all other studies reporting group interventions in children with DCD. The Bruininks-Oseretsky Test of Motor Proficiency (BOT-2) is also a product-oriented assessment that comprehensively evaluates motor competence in a variety of areas giving a detailed profile of children's motor skills. The Test of Gross Motor Development - 2e (TGMD-2) was selected because it is a process-oriented assessment that focuses uniquely on fundamental motor skills. The Beery-Buktenica Developmental Test of Visual-Motor Integration, 6e (Beery-VMI-6) taps into visual-motor integration: a combination of visual perception and motor coordination that applies to unique motor skills such as handwriting. Lastly, the Developmental Coordination Disorder Questionnaire (DCD-Q) was chosen as an assessment of guardian perception of a child's motor difficulties because it is the gold-standard for this type of evaluation in DCD. A detailed description of each assessment is provided below:

Movement Assessment Battery for Children, $2 e$ (MABC-2). This tool is a well-documented, individually-administered, standardized test that provides assessment for children with motor impairment. The MABC-2 is designed for children ranging from three to 16 years of age. In this study, Age band 2 ( 7 to 10 years) and 3 (11 to 16) were utilized. The test contains 8 subtests across 3 domains: Manual Dexterity, Aiming and Catching, and Balance. With good reliability (minimum value of test-retest at any age is .75, and inter-rater value is .70) and concurrent validity, it is frequently used to identify children with DCD. ${ }^{13}$ Typically, children obtaining scores below the $5^{\text {th }}$ percentile meet diagnostic criteria.

Developmental Coordination Disorder Questionnaire (DCD-Q). The DCD-Q is used as a parental report to screen for children with possible motor difficulties. This questionnaire contains 17 questions relating to the child's gross and fine motor skills. The scores range from 17 to 85 , and are categorized according to different cut-off points: 17 to 48 (DCD), 49 to 57 (suspected DCD), and 58 to 85 (probably not DCD). This tool has high internal consistency (alpha $=.94)$ and sensitivity $(85 \%) .11$

Bruininks-Oseretsky Test of Motor Proficiency (BOT-2). The BOT-2 covers the age range of 4 to 21 years, with a 53-item assessment consisting of eight subtests designed as game-like tasks that measure hand and arm coordination, balance, mobility, and strength. ${ }^{14}$ Four motor-area composites (Fine Manual Control, Manual Coordination, Body Coordination, and Strength \& Agility) are examined in order to create a Total Motor Composite (TMC) score which indicates overall motor abilities of the individual. All individuals were scored using combined norms, and each task was based on the child's preferred drawing hand, preferred throwing hand/arm, and preferred foot/leg. TMC reliabilities for the BOT-2 are in the mid .90s. There is an accumulation of evidence for the validity of the BOT-2, and it is strongly supported through both empirical and theoretical sources. ${ }^{11,15}$

Beery-Buktenica Developmental Test of Visual-Motor Integration, 6e (Beery-VMI-6). The Beery-VMI-6 is a norm-referenced standardized test, which is designed to measure visual-motor integration, the extent to which individuals can integrate their visual and motor abilities in individuals aged 2 to 100 years old. ${ }^{16}$ The supplemental tests of Visual Perception and Motor Coordination were also administered. The examinee is asked to copy 27 geometric forms, arranged in developmental sequence, from less to more complex. The designs range from very simple (a straight line) to quite complicated figures (cubes, overlapping circles). It is a commonly used standardized test with an objective scoring system. On the supplemental Visual Perception Test, the participants are asked to match the same 27 designs to the exact similar design among three to six alternatives. On the Motor Coordination Test, the participants are once again asked to draw the figures, but this time only by tracking within boundary lines delineating the correct design. Although the Visual Perception Test should measure the degree to which form perception contributes to overall VMI score, the Motor Coordination Test should measure the motor contribution. Standard scores, percentiles, and a descriptive category for each supplemental test and the total score were retrieved.

Test of Gross Motor Development - 2e (TGMD-2). The TGMD-2 is a well-validated standardized test that assesses fundamental motor skills of children between the ages of 3 and 10 years. ${ }^{17}$ The 12-item test includes two subscales: locomotor skills (run, gallop, hop, leap, horizontal jump, and slide) and object controls skills (striking a stationary ball, stationary dribble, catch, kick, overhand throw, and underhand roll). Each skill is evaluated on three to five performance criteria. If a participant demonstrates the criteria he/she received a 1 , if not a 0 . The totals for each skill were summed and represented the raw score (values range from 0 to 96 ).

(c) The Internet Journal of Allied Health Sciences and Practice, 2016 


\section{Procedures}

When guardians and children agreed to participate in the intervention, researchers scheduled two or three appointments (depending on the availability of the guardian/child) to complete the pre-test assessments within two weeks of the start of the program and were told they would schedule similar appointments within two weeks of the end of the program. The group motor intervention program consisted of activities that required collaboration among members and focused on activities that involved upper-body coordination, balance, and fine-motor skills and had common motor goals for the group. There were two intervention programs. The first, Program 1, offered a total of 24 sessions to participants, with sessions administered twice a week, for 12 weeks. Program 2 involved a total of 10 sessions administered once a week, for 10 weeks. Each session, regardless of the Program, lasted one hour. With Program 1, 6 sessions were designed to focus on each one of the components and the six final sessions combined upper-body coordination, balance, and fine-motor skills equally (total of 24). With Program 2, all 10 sessions combined the components equally. A detailed program was implemented and was based on developmentally appropriate characteristics of the participating children. An example of a session in Program 1 and 2 is described in Table 1. Every session started with a warm-up activity and was followed by a series of activities focusing on one of the motor components described above.

Table 1. Examples of Sessions in Program 1 and Program 2.

\begin{tabular}{|c|c|}
\hline Program & Activities \\
\hline $\begin{array}{l}1 \text { (24 sessions) } \\
\text { Example of a } \\
\text { session focusing } \\
\text { on "Balance" }\end{array}$ & $\begin{array}{l}\text { Acid river challenge: with "stones" made up of stepping stones, dome cones, and poly dots, children are to hold } \\
\text { hands/forearms and move across "stones" to designated finish line. Children decide the order of the line and can } \\
\text { reposition accordingly, monitor level of difficulty and challenge accordingly. } \\
\text { Balance pass: In a circle, children have a stepping stone under one foot and a dome cone under the other, and pass } \\
\text { a ball throughout the circle, with several objects introduced timely. Repeat with switch of stepping stone and dome } \\
\text { cone and if possible, challenge with both dome cones. } \\
\text { Poly dot pick up: Children stand on designated stepping stones (2) and dome cones (2) set on a diamond pattern, } \\
\text { and reach down to pick up poly dots around. They are allowed to drag stepping stones after they picked up all poly } \\
\text { dots around them, challenge of picking up with one leg only with the help of a partner. } \\
\text { Ball across the bridge: Children line up on both sides of laid out folded mats connected on the short sides to create } \\
\text { a long narrow bridge while they stand on two dome cones. Monitor rolls a gopher ball at one end of the mat bridge } \\
\text { and children have to reach down while standing on cones to gently roll the ball to another child, until the ball reaches } \\
\text { the end. Introduce more balls after they successfully complete one round. } \\
\text { Bear crawl path: Children spread stepping stones and dome cones around the room and have to bear crawl around } \\
\text { the path staying on the pattern, switch to crab walking when possible. Children are to move stones if they see a } \\
\text { friend needing help. } \\
\text { Volcano: Scatter poly dots around setup mats that look like a column, standing up right with an opening at the top } \\
\text { that can "hide" one person. With gopher balls and bean bags scattered, children are to collect one ball/bean bag at } \\
\text { a time and toss it inside the "volcano", walk on poly dots if necessary. Balls can only be tossed individually; bean } \\
\text { bags need a partner. The person inside the "volcano" continues to toss the balls/bean bags out of it to make it look } \\
\text { like a volcano. }\end{array}$ \\
\hline $\begin{array}{l}2 \text { (10 sessions) } \\
\text { Example of a } \\
\text { session combining } \\
\text { "Fine-motor skills, } \\
\text { balance, and } \\
\text { upper-body } \\
\text { coordination" }\end{array}$ & $\begin{array}{l}\text { Car and driver: In pairs, one child is blindfolded and the other is the driver, with his/her hands always on shoulders } \\
\text { of the "car." Driver is to guide the car at a slow walking pace around the room avoiding the other cars and other } \\
\text { objects throughout the room. Switch car and driver. } \\
\text { Group beading: In pairs, children bead first with pipe cleaners and then with a string, first one child picks up and } \\
\text { passes the bead to the other, then switches - use different grips and dominant/non-dominant hand. } \\
\text { Bean bag color toss: In groups of } 3 \text {, children are assigned a color. Padded mats are laid and attached to each other } \\
\text { so there is one long row of mats, with dome cones adjacent to the path. Mats have a color "assigned" to it. Children } \\
\text { throw bean bags only in their color cones and they can only throw from their color section at the mat. } \\
\text { Noodle bridge: Two children hold two noodles on the ends, facing each other. They hold and roll a ball back and } \\
\text { forth while taking it to the other side of the room. Continue until the ball basket is empty; add dumb cones for a } \\
\text { challenge. } \\
\text { Building: In a big group, children build something with small pipes. They have to work together to keep the pipes } \\
\text { connected. } \\
\text { Ball rolling: Two mats are set on opposite sides of room, squishy dome cones and stepping stones form a square in } \\
\text { the middle of the room. Two children roll a ball (can choose type) from each side, the goal is for the balls not to touch } \\
\text { until they hit the other side. Repeat with kicking. } \\
\text { Bubble wrapping: In pairs, children design a pattern on the bubble wrap and they can only pop two bubbles at a time, } \\
\text { then they have to switch. }\end{array}$ \\
\hline
\end{tabular}

All the activities had a common element: They involved the collaboration of two or more children. Children were verbally encouraged to cooperate and support each other and from watching and discovering new strategies to perform the skills. As 
proposed by Dunford, instructors suggested general problem solving strategies to the children based on task analysis and used scaffolding techniques. ${ }^{8}$ All instructors were pre-occupational therapy interns (senior undergraduate students) that were blind to the study parameters. They had at least a semester of experience working with children with DCD and were trained by the first author in the administration of the activities. Assistants were junior or senior pre-occupational therapy majors that helped the instructors by encouraging the participants and emphasizing the activities administered by instructors. Three instructors rotated in administering the intervention sessions while two aides assisted the children in performing the activities. All assessments were administered within a two week period before (pre-test) and after (post-test) the 24 sessions in Program 1 and the 10 sessions in Program 2. The pre- and post-test were administered in two sessions of about 3 hours each, with as many breaks as needed by the child, and were conducted at the first author's lab. All interventions took place in a large room of the University Recreation Center.

\section{Data Analysis}

Paired samples t-tests were conducted to detect differences between the pre- and post-test for all assessments (total scores and categories), for both standard scores and percentiles. T-tests were conducted separately for Program 1 and Program 2. For simplicity of presentation, all the results are presented using percentile scores (with the exception of the DCD-Q). When significant differences $(p<0.05)$ were indicated, the Cohen's $d$ estimated effect size analysis (for paired data) was calculated 18 to determine the practical significance of these differences, where a $d$-value of $>0.3$ indicates a small effect size, $>0.5$ a moderate effect size and $>0.8$ shows a large effect size.

\section{RESULTS}

Table 2 shows means and standard deviation values for all tests in Programs 1 and 2.

Table 2. Means and standard deviations for pre- and post-test scores in Program 1

(24 sessions) and Program 2 (10 sessions)

\begin{tabular}{|c|c|c|c|c|}
\hline & \multicolumn{2}{|c|}{ Program $1(n=8)$} & \multicolumn{2}{|c|}{ Program $2(n=6)$} \\
\hline & Mean (SD+) & Mean $(S D+)$ & Mean $(S D+)$ & Mean (SD+) \\
\hline Assessment/Component & Pre-test & Post-test & Pre-test & Post-test \\
\hline \multicolumn{5}{|l|}{ MABC-2 } \\
\hline - Manual Dexterity & $6.9(11.1)$ & $3.7(3.8)$ & $21.3(27.4)$ & $19.8(27.3)$ \\
\hline - Aiming \& Catching & $6.7(6.2)$ & $13.9(19.2)$ & $20.4(20.2)$ & $19.1(28.1)$ \\
\hline - Balance & $5.7(8.6)$ & $10.8(18)$ & $8.9(8.8)$ & $7.8(14.4)$ \\
\hline - Total Score & $2.6(5.4)$ & $4.2(8.4)$ & $6.1(6.2)$ & $12(24.9)$ \\
\hline \multicolumn{5}{|l|}{ BOT-2 } \\
\hline - Fine Manual Control & $7.2(4.9)$ & $14.7(13.9)$ & $22(18.7)$ & $28.4(33.3)$ \\
\hline - Manual Coordination & $12.5(14.6)$ & $17.8(15.6)^{*}$ & $31.8(19.4)$ & $45.4(29.5)$ \\
\hline - Body Coordination & $5.1(3.3)$ & $16.7(17)$ & $17.8(10.2)$ & $23(17.8)$ \\
\hline - Strength \& Agility & $16.1(10.3)$ & $21(18.4)$ & $25.2(19.9)$ & $28(16.5)$ \\
\hline - Total Motor Composite & $5.6(2.7)$ & $11.5(7.1)^{*}$ & $17.5(5.1)$ & $15.5(13.4)$ \\
\hline \multicolumn{5}{|l|}{ Beery-VMI-6 } \\
\hline - Visual Perception & $39.4(38.4)$ & $37.1(33.7)$ & $39.5(35.4)$ & $56(29.8)$ \\
\hline - Motor Coordination & $17.9(26.6)$ & $31.2(27.9)$ & $34(35.6)$ & $32.5(31.6)$ \\
\hline - Visual-Motor Integration & $20.5(18.2)$ & $24.9(20.2)$ & $43.6(31.3)$ & $52.5(30.5)$ \\
\hline \multicolumn{5}{|l|}{ TGMD-2 } \\
\hline - Locomotor Skills & $14.4(13.7)$ & $22.6(20.32)$ & $31(8.4)$ & $37.5(17.6)$ \\
\hline - Object Control & $4(6.7)$ & $18.6(20.3)$ & $33(24.04)$ & $44(26.8)$ \\
\hline - Gross Motor Quotient & $18.6(39.3)$ & $17.6(19.4)$ & $22(28.2)$ & $38.5(4.9)$ \\
\hline \multicolumn{5}{|l|}{ DCD-Q } \\
\hline - Control during Movement & $12(1.5)$ & $16.1(4.2)^{\star *}$ & $14.4(4)$ & $13(5.5)$ \\
\hline - Fine motor/Handwriting & $7.1(1.1)$ & $9.1(2.2)^{*}$ & $7(2)$ & $6.8(1.9)$ \\
\hline - General Coordination & $10.5(2.7)$ & $12.6(3.1)^{*}$ & $9.2(4.3)$ & $9.6(5.3)$ \\
\hline - Total Score & $26.8(5.1)$ & $38.3(7.6)^{*}$ & $30.6(9.7)$ & $29.4(11.5)$ \\
\hline
\end{tabular}

* Significant change in paired t-test, $p<.05$

** Significant change in paired t-test, $p<.01$

Program 1 (24 one hour sessions- twice a week for 12 weeks)

Program 2 (10 one hour sessions- once a week for 10 weeks) 
MABC-2. No significant changes were found for each component (Manual Dexterity, Balance, Aiming and Catching) or the Total Score $(p s>.05)$ for both programs.

\section{BOT-2.}

Program 1. One child did not complete the post-test for the BOT. T-test analysis revealed significant improvements in the percentile scores for the components Manual Coordination, $\mathrm{t}(6)=-3.58, p<.02$ and the Total Motor Composite, $t(5)=-2.98, p<.04$. The Fine Manual Control and Strength \& Agility Component were not significantly different $(p>.05)$. The Cohen's $d$ effect sizes were .3 for Manual Coordination and 1.0 for Total Motor Composite.

Program 2. No significant differences were found in any component or Total Motor Composite.

Beery-VMI-6. No significant differences were indicated in Visual Perception, Manual Coordination, and Visual-Motor Integration ( $p$ $>$.05).

DCD-Q.

Program 1. Parents reported significantly higher scores for all categories of the questionnaire, Fine Motor/ Handwriting, $\mathrm{t}(7)-2.82$, $p<0.5$, Control during Movement, $\mathrm{t}(7),-3.83, p<.01$, General Coordination, $\mathrm{t}(7)=-3.06, p<.05$, and Total Score, $\mathrm{t}(7)=-2.75, p$ $<.05$. The Cohen's d effect sizes were 1.1 for Fine Motor/Handwriting, 1.3 for Control during Movement, .7 for General Coordination, and 1.7 for Total Score.

Program 2. No significant differences were found in any category or Total Score.

TGMD-2. No significant differences in Locomotor, Object Control, and Gross Motor Quotient were found ( $p>$.05).

\section{DISCUSSION}

The purpose of this study was to determine the effectiveness of a group intervention program for children with $D C D$ and to compare whether frequency of each program (10 one hour sessions - once/week verse 24 one hour sessions - twice/week of intervention) resulted in different motor performance outcomes. The results confirmed the hypothesis- while guardian perception (as measured by the DCD-Q) and some aspects of motor proficiency (Manual Coordination and Total Motor Proficiency, as measured by the BOT-2) improved after Program 1 (24 one hour sessions- twice a week for 12 weeks), no significant changes were found in Program 2 (10 one hour sessions- once a week for 10 weeks). After Program 1, (24 one hour sessions- twice a week for 12 weeks), parental perception of their child's difficulties improved in all aspects (Control during Movement, Fine motor/Handwriting, and General Coordination), potentially indicating that some daily/functional activities may have improved throughout the program. At this point, it is unclear whether the changes observed are due to skill/ability transfer (from the group activities to individual actions) or secondary possible changes in confidence, self-esteem, or any other psychological aspects that may have changed with the program (and not measured). Possibly it is a combination of both. While aspects of the group collaboration may have boosted a child's overall well-being, it is possible that the volume of motor training provided by Program 1 (10 one hour sessions- once a week for 10 weeks) enabled their performance and provided strategies to accomplish motor skills.

In reference to the improvement noted by categories and the total score on the Bruininks-Oseretsky Test of Motor Proficiency and no other assessments, the changes seen may be due to the nature of the test. The BOT-2 is categorized as a product-based assessment, where the outcome of the action is more important than the quality of the movement. The Manual Coordination category is subdivided into Upper-limb coordination and Manual dexterity abilities. The upper-limb coordination subsection has no time constraints and involved items such as dropping and catching a ball, catching a tossed ball, dribbling and throwing. While the lack of time constraints and no limit on the number of trials a child is given to possibly reach full score may have helped children accomplish tasks successfully, ball skills were a major focus of the programs, and working with children who had similar difficulties with ball skills may have aided with the improvement of this category. Conversely, the manual dexterity subsection has time constraints and therefore can be considered more difficult for children with DCD. However, working as a group may have facilitated the development and learning of new strategies from other children, which could have aided the improvement of this category after Program 1(10 one hour sessions- once a week for 10 weeks).

The other assessments indicated no improvements in their respective areas (gross motor skills, visual-motor integration), and may be due to the nature of the assessments and the intervention completed. It is possible that some of the skills measured by the other assessments can only be improved with more focused, individual interventions. While the children may have developed different strategies to accomplish certain tasks required by the BOT-2 assessment, they have not improved qualitative patterns required by other assessments, especially the TGMD-2. These findings support the idea that task-oriented approaches for intervention with children with DCD yield stronger effects than process-oriented approaches. ${ }^{4} \mathrm{As}$ indicated earlier, the goal of the intervention was to have the children complete the activities with the group, and not to improve specific qualitative patterns of motor

(C) The Internet Journal of Allied Health Sciences and Practice, 2016 
skills within the activities.

Looking at skills tapped by the BOT-2 assessment, it appears that some of the skills may have been transferred to performance in daily living activities as measured by parental response with the DCD-Q. The goal of this questionnaire is to have parents rate their children's functioning in Control during Movement, Fine Motor/Handwriting, and General Coordination items compared to other children the same age as theirs. While the program sessions did not focus on establishing generalization and transfer of motor skills to the items of the DCD-Q, it is possible that the improvement in parent perception is related with skills practiced in the program. There is also the likelihood that the group approach helped increase peer support among families, helping guardians to notice improvements in their children's motor performance.

It seems that frequency of the program played a fundamental role in a child's improvement. Positive changes seen after a 24session program were not seen after a "shorter" program with only 10-sessions. The previous studies used a shorter number of sessions; in eight and ten sessions. 5,6 Dunford noted that scheduling of concentrated and intensive practice combined with group intervention was associated with significant improvements in motor impairment and goal attainment. ${ }^{8}$ This author utilized intensive scheduling, with eight sessions over a 2-week period, and improvements were noted. It appears that children with DCD may benefit from more sessions in an intervention program to demonstrate improvement. Differences in the focus of the intervention may be relevant as well. Dunford's study focused on goal achievement, as did this study with the added dimension of working with peers. ${ }^{8}$

\section{LIMITATIONS}

This study has several limitations. The small sample size and lack of group randomization were a limitation. The participants selfselected the group intervention they felt they could commit to. The method of intervention, although followed similar guidelines, had some variations, with the 24 session program specifically focusing on skill development, while the 10-session intervention had a combined focus (due to the shorter number of sessions). Possibly the specific skills training may have been a factor.

\section{FUTURE RESEARCH}

More research is needed to confirm those results, address the limitations of this study, and to explore further the notion of frequency, intensity, and duration of group interventions in children with DCD. The promotion of group interventions can provide the option for offering more sessions (and more often) to the same child, which can be beneficial for the improvement of general motor abilities and skills. In addition, it is necessary to explore other aspects that emerge from a group setting, such as social interaction and perceived competencies. It is possible that group interventions will provide similar effects as individual interventions, but may enhance the outcomes by promoting other social and psychological components that not offered individually. It appears that group interventions are a promising method of service delivery. ${ }^{19}$

\section{CONCLUSION}

We conclude that a group intervention setting that focuses on activities involving collaboration among members of the group and a significant frequency (at least twice a week) can be an effective intervention method for improvement of motor performance in children with Developmental Coordination Disorder. This study illustrates several aspects of clinical relevance. First and foremost, it provided evidence that children with DCD can benefit and improve motor skills after a group intervention. This finding enables therapists to address long waiting lists by grouping children for intervention sessions, and also increase the number of intervention sessions promoted for children with DCD. Because frequency was an important factor in improvement, professionals are encouraged to find possible alternatives to provide a higher number of sessions for these children. In addition, this study reinforced the current evidence that emphasizes that task-oriented approaches are beneficial for children with DCD. Allied health professionals and therapists should consider this approach as a feasible intervention method to improve product-oriented motor skills in children.

\section{REFERENCES}

1. American Psychiatric Association. Diagnostic and Statistical Manual of Mental Disorders, 5e. Washington: American Psychiatric Association, 2013.

2. Vaivre-Douret L, Lalanne C, Ingster-Moati I, Boddaert N, Cabrol D, Dufier J, Golse B, Falissard B. Subtypes of Developmental Coordination Disorder: Research on their nature and etiology. Dev Neuropsychol. 2011;36(5):614-43. [PMID 21667364]

3. Hillier S. Intervention for children with developmental coordination disorder. A systematic review. IJAHSP. $2007 ; 5: 3$.

4. Smits-Engelsman BC, Blank R, van der Kaay AC, Mosterd-van der Meijs R, Vlugt-Vvan den Brand E, Polatajko HJ, Wilson $\mathrm{PH}$. Efficacy of interventions to improve motor performance in children with Developmental Coordination Disorder: A combined systematic review and meta-analysis. Dev Med Child Neurol. 2013 Mar;55(3):229-37. [PMID 23106530]

(C) The Internet Journal of Allied Health Sciences and Practice, 2016 
5. Pless M, Carlsson M. Effects of motor skill intervention on Developmental Coordination Disorder: A meta-analysis. Adapt Phys Activ. 2000;17:381-401.

6. Pless M, Carlsson M, Sundelin C, Persson K. Effects of group motor skill intervention on five- to six-year-old children with Developmental Coordination Disorder. Pediatr Phys Ther. 2000 Winter;12(4):183-9. [PMID 17091030]

7. Hung WW, Pang MY. Effects of group-based versus individual-based exercise training on motor performance in children with Developmental Coordination Disorder: A randomized controlled trial. J Rehabil Med. 2010 Feb;42(2):122-8. [PMID 20140407]

8. Dunford C. Goal-orientated group intervention for children with Developmental Coordination Disorder. Phys Occup Ther Pediatr. 2011 Aug;31(3):288-300. [PMID 21488710]

9. Henderson SE, Sugden DA, Barnett AL. Movement Assessment Battery for Children,2e. San Francisco, CA: Pearson, 2007.

10. Palisano RJ, Murr S. Intensity of therapy services: What are the considerations? Phys Occup Ther Pediatr. 2009;29(2):10712. [PMID 19401925]

11. Wilson BN, Kaplan BJ, Crawford SG, Campbell A, Dewey D. Reliability and validity of a parent questionnaire on childhood motor skills. Am J Occup Ther. 2000;54(5):484-93. [PMID 11006808]

12. Kaufman AS, Kaufman NL. Kaufman Brief Intelligence Test,2e. Bloomington, IN: Pearson, 2004.

13. Zwicker JG, Missiuna C, Harris SR, Boyd LA. Developmental Coordination Disorder: A review and update. Eur J Paediatr Neurol. 2012 Nov;16(6):573-81. [PMID 22705270]

14. Bruininks RH, Bruininks BD. Bruininks-Oseretsky Test of Motor Proficiency,2e. Minneapolis, MN: Pearson, 2005.

15. Düger T, Bumin G, Uyanika M, Aki E, Kayihan H. The assessment of Bruininks-Oseretsky test of motor proficiency in children. Pediatr Rehabil. 1999;3:125-31.

16. Beery KE, Buktenica NA, Beery NA. Beery-Buktenica Developmental Test of Visual-Motor Integration,6e. San Antonio, TX: Pearson, 2010.

17. Ulrich D. Test of Gross Motor Development,2e. Austin, TX: Pro-Ed, 2000.

18. Son SH, Morrison FJ. The nature and impact of changes in home learning environment on development of language and academic skills in preschool children. Dev Psychol. 2010;46(5):1103-18. [PMID 20822226]

19. Camdem C, Tétreault S, Swaine B. Increasing the use of group interventions in a pediatric rehabilitation program: Perceptions of administrators, therapists, and parents. Phys Occup Ther Pediatr. 2012 May;32(2):120-35. [PMID 21942928] 